\title{
Estimation of the Cell Loss Ratio in ATM Networks with a Fuzzy System and Application to Measurement-Based Call Admission Control
}

\author{
Brahim Bensaou†, Shirley T. C. Lam $\ddagger$ Hon-Wai Chuł, and Danny H. K. Tsang
}

\begin{abstract}
An important parameter in ATM-based network design and management is the cell loss ratio (CLR) in ATM multiplexers. It is a key parameter to many vital functions in the network such as call admission control, bandwidth allocation, etc. However, the CLR depends usually on many unknown and unpredictable traffic parameters such as input traffic correlations. In this paper, we propose a simple and robust fuzzy-based algorithm to predict the CLR in large-sized systems based on both a small amount of information from small-sized systems, and the asymptotic behavior for very large systems. Unlike the model-based approaches, our approximation avoids the problem of assuming any traffic parameters or arrival process. This algorithm is used with real-time traffic measurement to propose an effective measurement-based call admission control framework for ATM networks.
\end{abstract}

Keywords - ATM, cell loss ratio, fuzzy logic, call admission control.

\section{INTRODUCTION}

An asynchronous transfer mode (ATM)-based Broadband Integrated Services Digital Network (BISDN) is a single high speed transport network designed to support all known and unknown services with different quality of service (QoS) requirements. It transfers the information through fixed size packets called cells. The network takes advantage of the statistical behavior of the sources with different traffic characteristics to efficiently share transmission resources through statistical multiplexing. ATM technique is connection-oriented (i.e. a connection must be established before the actual data exchange can begin). On connection request, a contract is established between the network and the source. The source first declares a set of parameters to describe its traffic and agrees to generate its traffic according to the declared parameters. Once the call is accepted, the network agrees to guarantee the required QoS. The QoS is generally specified

This work was done while Dr Bensaou was Research Associate with the Department of Electrical and Electronic Engineering of HK University of Science and Technology

tCenter for Wireless Communication, Singapore Science Park II, 117674, Singapore. E-mail: cwcbb@nus.sg $\ddagger$ Department of Electrical and Electronic Engineering The Hong Kong University of Science and Technology, Hong Kong. E-mail: \{lamtc, eechu, eetsang\}@ee.ust.hk in terms of end-to-end cell delay, and cell loss ratio in the ATM multiplexers. An ATM multiplexer is commonly modelled as a buffer of size $K$ (cells) and a single output link with a transmission capacity of $C$ cells per second. When cells arrive to the multiplexer at a rate greater than the transmission capacity while the buffer is full, cell loss occurs. To allow the network to decide whether to accept a new connection or not, given the available transmission resources, an accurate estimation of the cell loss ratio (CLR) is important. Besides call admission control (CAC), an accurate estimation of the CLR in ATM multiplexers is of paramount importance to other network management procedures such as network resources dimensioning (e.g. buffer dimensioning), congestion control (e.g. traffic monitoring), routing, etc. Throughout this paper, unless stated otherwise, we will use the acronym CLR to refer to either the cell loss ratio, the cell loss probability, or the overflow probability, depending on the model we use.

Many approaches for evaluating the CLR at the so-called burst-scale congestion for multiplexers loaded with superposition of on/off sources have been proposed in the literature. The most attractive approach, because of its mathematical tractability, approximates the actual arrival process to the buffer by a fluid flow (FF) [1], [2], [3]. In this approximation, the fluctuation of the cell arrival rates can be represented accurately by assuming that the information arrives in a continuous flow rather than in discrete cells. In particular, when the rate fluctuation occurs at a much larger time scale than the cell inter-arrival times, the effects on the CLR due to the discrete nature of the actual arrival process become negligible. The CLR is approximated accurately by the overflow probability which is obtained by solving an adequate system of differential equations, leading under certain (common) assumptions to a closed form solution [1] or under more general assumptions to a numerical solution (e.g. [3], [2]). Note that the cell loss probability can also be obtained; however this can be achieved at the expense of complex, time-consuming numerical 
algorithms [4]. Another attractive approach, very similar to the fluid flow approach, approximates the actual arrival process by a Markov Modulated Deterministic Process (MMDP) in which cells arrive according to a deterministic renewal process whose rate is controlled by a Markov process [5]. The attractive feature of this approach is that unlike the FF approach, it leads to the exact cell loss probability by solving a set of linear equations through a Gauss-Seidel algorithm. Many other approximations, such as MMPP, are also available; however, due to their analytical and numerical complexity, they are not appealing from the practical point of view.

These approaches are efficient in predicting the CLR in an ATM multiplexer. However, when the system size becomes large, computation complexity increases in the FF approximation and memory problems arise in the MMDP and MMPP approximations. It is also well known that the CLR depends on many unknown and unpredictable traffic parameters, such as burst and silence lengths distributions [3] and more generally, long term correlations [6].

To avoid the problems of the previously introduced methods, other approximate approaches have also been proposed in the literature to estimate the CLR in ATM multiplexers. For example, in [7] approximations have been derived for the queue length distribution of a fluid multiplexer fed by a superposition of homogeneous on/off sources. This approach uses the large deviation estimates of the queue length distribution obtained when the link capacity and the multiplexer buffer size scale (or diverge) with the number of sources sharing the multiplexer. In [8] the same approach is used to derive the large deviation estimates of the queue length distribution for more general heterogeneous arrival processes such as superposition of Gaussian processes, Ornstein-Uhlenbeck processes, etc. Although the large deviations estimates give very good approximations of the CLR even under more practically interesting assumptions (e.g. reasonably small number of sources) while avoiding the complexity of the FF, MMDP, or MMPP models, they still depend on the knowledge of the arrival process, which for most practical ATM sources is yet to be determined. For instance, although MPEG video sequences have been shown to exhibit a self-similar behavior, it is shown in [9] that there is no typical video source behavior (i.e., the parameter characterizing the self-similarity changes from one type of video application to another and even from one video source to another).

Due to these kinds of restrictions, our goal is to develop a new, simple and practical algorithmic approach to estimate the CLR in large-sized sys- tems, relying only on information from the smallsized systems and some qualitative information on the CLR function. Depending on the problem under consideration, the system size can be either the multiplexer buffer size, the number of connected users or the link capacity. Our proposed approach takes advantage of the qualitative and quantitative information provided by analytical models and/or real time measurements, without suffering from the commonly made simplifying assumptions in analytic modelling or prohibitively long measurement times.

In the same spirit, the Global Rational Approximation (GRA) algorithm in [10] is proposed to estimate the cell loss probability. In this method, the CLR as a function of buffer size (or of number of users) is approximated by a rational function, of the form $R(x)=P_{m}(x) / Q_{n}(x)$ where $P_{m}$ and $Q_{n}$ are polynomial functions of degree $m$ and $n$ respectively. The coefficients of $P_{m}$ and $Q_{n}$ are determined by solving the system of linear equations: $R\left(x_{i}\right)=\operatorname{Pr}\left(x_{i}\right), i=1, \cdots, m+n$, where the pairs $\left(\operatorname{Pr}\left(x_{i}\right) ; x_{i}\right)$ represent the small-sized system information (e.g. (CLR; buffer size), (CLR; number of users))[10].

Even though the GRA approximation is efficient and accurate in many cases, it still has some major problems:

- the accuracy of the approximation is closely related to the degrees $m$ and $n$, which in turn determine the number of pairs $\left(\operatorname{Pr}\left(x_{i}\right) ; x_{i}\right)$ required (i.e., $m+n$ pairs). When $m$ and $n$ are not large enough, the accuracy of GRA can be poor, and

- the computation time can sometimes be quite long since the fitting process is based on an iterative algorithm to determine the suitable values of $m$ and $n$ for the required accuracy. In addition, depending on the values of the pairs $\left(\operatorname{Pr}\left(x_{i}\right) ; x_{i}\right)$, the system of equations to solve can sometimes encounter singularity problems, which make the fitting process difficult. We noticed that this problem occurred mainly when the known values of CLR do not fit on a smooth curve, which is the case when the known values of CLR are obtained from measurements that normally contain a small amount of error.

Fuzzy logic technique has been used to efficiently solve several ATM problems such as traffic enforcement [11], traffic control [12], [13], congestion control in ABR service [14] and routing [15]. Thus, in view of the shortcomings encountered in the above mentioned approaches, we propose to use an adaptive fuzzy system to predict efficiently the CLR in large-sized systems based on a small amount of information from small-sized systems. This information can be obtained by either real-time measure- 
ment when the traffic characteristics are unknown or from any analytic model when the traffic characteristics are given. This predicted CLR is then refined, by taking advantage of the knowledge of the asymptotic behavior of the performance curve (cell loss ratio). The advantages of using the proposed fuzzy logic based algorithm are mainly the simplicity in implementation, the computational efficiency, and good accuracy.

The paper is organized as follows. In Section 2, we present a brief introduction to an adaptive fuzzy system and then describe the proposed fuzzy approximation (FA) algorithm. In Section 3, the proposed algorithm is applied to predict the CLR as a function of the buffer size, and the multiplexer service capacity respectively. To show the accuracy of the predictions, some numerical examples are given for each case and comparison to the results obtained from alternative methods are also shown. The independence of our algorithm from any assumptions on the input traffic characteristics is given in this section. Based on this FA algorithm, we propose in Section 4 a practical call admission control algorithm for ATM networks. In particular, the FA algorithm is implemented as a part of a more complex CAC mechanism where the small-sized information is obtained from real-time measurement by using a set of virtual buffers (counters) with reduced service capacity. Finally, we present our conclusions in Section 5.

\section{Fuzzy Approximation (FA)}

In this section, we briefly introduce fuzzy logic and adaptive fuzzy systems and show how they can be used to represent an unknown mathematical model. We will then present how this type of systems is applied to predict the CLR in a largesized system by taking information from both the small-sized systems and the asymptotic behavior of the performance curve for the large-sized system. We remind the reader that the fuzzy logic theory and how fuzzy systems are constructed are beyond the scope of this paper; more comprehensive and simple presentations on fuzzy sets theory can be found in [16], [17]. In the paragraphs that follow, we will focus mainly on the practical issues, such as the estimation of the CLR and how to use the fuzzy algorithm to develop a CAC mechanism in ATM networks.

\section{A. Overview of fuzzy rule-based systems}

Fuzzy systems, unlike what the "non-initiated" person thinks, are precisely defined with a strong mathematical basis. What is "fuzzy" in a fuzzy system is the information it deals with. The fuzzy sets theory describes incomplete or vague concepts which might be difficult to formulate mathemati-

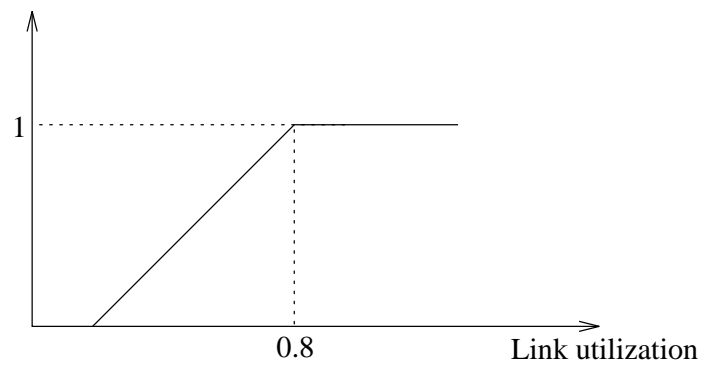

Fig. 1. Membership function of the linguistic variable "close to congestion"

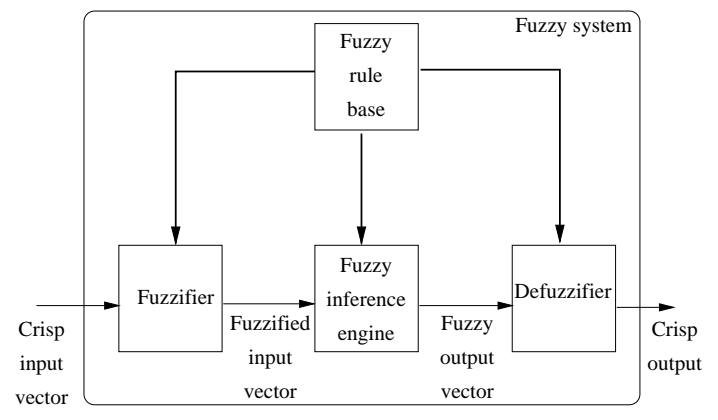

Fig. 2. Block diagram of a fuzzy system

cally. Fuzzy systems are knowledge-based systems (also called rule-based systems). The heart of a fuzzy system is a knowledge base which consists of a set of IF-THEN rules. The rules are statements in which some words are characterized by continuous membership functions. For example, in the following IF-THEN rule:

$$
\begin{aligned}
& \text { IF the link is close to congestion } \\
& \text { THEN reduce the input rate, }
\end{aligned}
$$

the words close to congestion are characterized by a membership function as shown in Figure 1, where congestion is considered happening when the link utilization is above 0.8 . The word reduce can also be characterized by another membership function.

A fuzzy system is basically made of a fuzzifier, a defuzzifier, an inference engine and a rule base as shown in Figure 2. The role of the fuzzifier is to map the crisp input data values to fuzzy sets defined by their membership functions depending on the degree of "possibility" of the input data. There are many types of fuzzifiers among which, the most commonly considered in practical applications is the singleton fuzzifier which maps the crisp input to a singleton fuzzy set. Intuitively, this means the degree of possibility of the input data is equal to 1. When the degree of possibility of the input data can only be known in a certain range, other fuzzifiers such as the Gaussian or triangular are also available. In this paper, the singleton fuzzifier is chosen to implement our fuzzy system. The goal of 
the defuzzifier is to map the output fuzzy sets to a crisp output value. It combines the different fuzzy sets with different degrees of possibility to produce a single numerical value. The most commonly used defuzzifier is the center-average defuzzifier which calculates a weighted average of the centers of the output fuzzy sets to produce the crisp output. This defuzzifier is chosen to implement our fuzzy system. The fuzzy inference engine defines how the system should infer through the rules in the rule base to determine the output fuzzy sets. Without going into details, which are beyond the scope of this paper, many inference engines are available. To construct our system, the product inference engine is chosen.

Suppose we have a system whose behavior is difficult to represent accurately by analytical models, for instance, an ATM multiplexer queue whose performance depends on the input traffic which in turn is unpredictable. Furthermore, suppose we know $N$ sets of input-output pairs $\left(\mathbf{X}_{0}^{j} ; y_{0}^{j}\right)$, $\mathbf{X}_{0}^{j}=\left(x_{01}^{j}, \ldots, x_{0 n}^{j}\right)^{\mathrm{T}} \in R^{n}, j=1,2, \ldots, N$, where $N$ is a small number. In [17], it is shown that fuzzy systems with singleton fuzzifier, product inference engine and centre-average defuzzifier reduce to the compact form

$$
f(\mathbf{X})=\frac{\sum_{j=1}^{N} y_{0}^{j} \prod_{i=1}^{n} \mu_{i}^{j}\left(x_{i}\right)}{\sum_{j=1}^{N} \prod_{i=1}^{n} \mu_{i}^{j}\left(x_{i}\right)}
$$

where $\mathbf{X}=\left(x_{1}, \ldots, x_{n}\right)^{\mathrm{T}} \in R^{n}$ is the (crisp) input to the fuzzy system, $f(\mathbf{X}) \in R$ is the output of the fuzzy system, and $\mu_{i}^{j}$ are the membership functions of the input fuzzy sets. It is also shown in [17] that if in addition the membership functions $\mu_{i}^{j}$ are Gaussian (i.e. $\mu_{i}^{j}\left(x_{i}\right)=\exp \left\{-\left(x_{i}-x_{0 i}^{j}\right)^{2} /\left(\sigma_{i}^{j}\right)^{2}\right\}$, $j=1, \ldots, N, i=1, \ldots, n)$, then (2) has universal approximation capabilities. That is, for any given real continuous function $g(\mathbf{X})$ and arbitrary $\epsilon>0$, there exists a fuzzy system in the form of (2) such that $\sup _{\mathbf{X} \in R^{n}}|f(\mathbf{X})-g(\mathbf{X})|<\epsilon$. This justifies our choice of the singleton fuzzifier, product inference engine and center average defuzzifier with Gaussian membership functions to implement our fuzzy system.

Usually, when using (2) for interpolating functions, the smaller the value of $\sigma_{i}^{j}$, the better is the accuracy of the approximation. However, for extrapolating a function, which is our objective, the approximation error cannot be controlled theoretically. In this case, the values of $\sigma_{i}^{j}$ should be chosen such that the corresponding membership function uniformly covers all the $n$ components of the $j^{t h}$ input to ensure that the influence of each of the $n$ components is reasonably significant.

\section{B. Estimation of the CLR}

The fuzzy approximation (FA) we propose to estimate the CLR in ATM multiplexers is based on the above fuzzy system. We view the CLR as an unknown function of a real variable which can be the multiplexer's buffer size, the number of connections or the service capacity.

The algorithm we propose to predict the CLR does not assume anything about the traffic parameters but requires only the knowledge of the following:

1. the CLR of the multiplexer when the system size is small (e.g. small buffer size, or small service capacity). In this case, the CLR is relatively large and can be calculated or measured quickly and easily;

2. the asymptotic behavior of the CLR when the system size is very large (e.g. infinitely large buffer size or reasonably large service capacity).

Let $P(m), m=1,2, \cdots$, denote the cell loss ratio when the system is of size $S_{m}$, where $S_{m}$ can be the buffer size, the number of users or the service capacity. The set of values $\left(S_{m}\right)$ should define a linearly increasing function of the form: $S_{m}=m \delta+$ $S_{0}$, where $\delta>0$ is the step size and $S_{0}$ is the initial value.

Based on the above set of information, the FA algorithm is constructed from $M$ known values of the CLR, $P(k-M+1), P(k-M+2), \ldots, P(k)$, where $k$ is the index of the current state $S_{k}$, as follows:

Step 1: Input-output pairs definition

The $(M-2)$ sets of input-output pairs are chosen as follows:

$$
\begin{aligned}
& \{(P(k-2), P(k-1)) ; P(k)-P(k-1)\} \\
& \{(P(k-3), P(k-2)) ; P(k-1)-P(k-2)\} \\
& \vdots \\
& \{(P(k-M+1)), P(k-M+2)) ; \\
& \quad P(k-M+3)-P(k-M+2)\}
\end{aligned}
$$$$
\text { : }
$$

where $(P(k-j-1), P(k-j))$ is the input vector and $P(k-j+1)-P(k-j)$ is the output for $j=$ $1, \ldots, M-2$.

By taking advantage of the monotonicity of the parameterized curve, which represents the CLR as function of the buffer size, the service capacity or the number of calls, the fuzzy system can be constructed from only a few $(M-2)$ input-output pairs. The inputs are represented by the CLR values from the previous and the current states. The output is the difference between the next and the current CLR values. The input-output pairs are updated in real time when $k$ is incremented toward the desired value.

Step 2: Membership functions 
Define $\Delta_{k, j}$ as $\Delta_{k, j}=(P(k-1)-P(k-j-1)$. For $j=1, \ldots, M-2$, the membership functions of the input fuzzy sets are then defined as [18]:

$$
\begin{cases}\mu_{1}^{j}(P(k-1)) & =\exp \left\{-\frac{\Delta_{k, j}^{2}}{\left(\sigma_{1}^{j}\right)^{2}}\right\}, \\ \mu_{2}^{j}(P(k)) & =\exp \left\{-\frac{\Delta_{k+1, j+1}^{2}}{\left(\sigma_{2}^{j}\right)^{2}}\right\},\end{cases}
$$

with

$$
\left\{\begin{aligned}
\sigma_{1}^{j} & =\frac{\max _{j} P(k-j-1)-\min _{j} P(k-j-1)}{i-2}, \\
\sigma_{2}^{j} & =\frac{\max _{j} P(k-j)-\min _{j} P(k-j)}{i-2} .
\end{aligned}\right.
$$

As mentioned above, they define the degree of influence of the first (respectively the second) component of the input vector $j$ on the output value. Note that $\sigma_{1}^{j}$ and $\sigma_{2}^{j}, j=1, \ldots, M-2$, are free parameters that determine the accuracy of the approximation. We choose $\sigma_{1}^{j}$ and $\sigma_{2}^{j}, j=1, \ldots, M-2$, as above to make the membership functions uniformly cover the range of the inputs vectors. In other words, the influence of the previous values of the CLR on the next predicted value is uniform.

Step 3: Fuzzy system construction

The system we use is obtained from (2) by replacing $n=2$ and $N=M-2$. As shown in Step 1, the output of the fuzzy system is defined as the increment (or decrement) of the CLR with respect to the previous value. At the beginning, the values of the state variable for which the CLR is known are indexed as $0, \ldots, M-1$. Equation (2) is first used to estimate the CLR for the next state variable (i.e. for $S_{M}$ ). When the CLR is obtained, the oldest point $S_{0}$ is dropped and the newly estimated point is fed back to the system. In other words, the states indexed by $1, \ldots M$ are used to construct the inputoutput pairs needed to define the new fuzzy system to estimate the CLR for $S_{M+1}$. This process continues until either the target CLR is reached or the CLR for the desired size of the system is obtained.

Generally, the FA algorithm provides an accurate prediction when the number of steps required to predict the target CLR is not too large. Nevertheless, predicting CLR values as small as $10^{-10}$ sometimes requires a large number of steps. This makes the accuracy of the prediction poor because of the accumulation of small prediction errors. In order to improve the estimation, we propose to take into account the asymptotic behavior of the CLR as the system size $S_{m}$ becomes large.

Let $\hat{P}(m)$ be the value of the CLR predicted by the FA algorithm, and $\tilde{P}(m)$ be the value obtained from the asymptotic behavior of the CLR as a function of the system size $S_{m}$. We propose to replace the predictions fed back to the FA algorithm in Step 3 above by the following approximation

$$
P(m)=(1-\lambda(m)) \hat{P}(m)+\lambda(m) \tilde{P}_{2}(m),
$$

where $\lambda(m)$ is a nonlinear increasing function of $S_{m}$ taking its values in $[0,1]$.

The function $\lambda(m)$ we use is given by

$$
\lambda(m)= \begin{cases}\exp \left(-\frac{\left(S_{m}-S_{\infty}\right)^{2}}{\sigma^{2}}\right) & \text { if } S_{m} \leq S_{\infty} \\ 1 & \text { elsewhere }\end{cases}
$$

where $S_{\infty}$ is any state at which the asymptotic behavior holds and $\sigma$ is a parameter controlling the accuracy of the approximation. The function $\lambda(\cdot)$ introduced above acts as a high pass filter. It enables the influence of the asymptotic behavior only when the system size $S_{m}$ is close to $S_{\infty}$. The determination of $S_{\infty}$ and $\sigma$ is discussed later in the next section.

\section{Numerical Examples and Discussions}

The implementation of the algorithm described above proves straightforward, and the CPU time needed to predict the CLR is quite small. The complexity of the FA algorithm is a linear function of the number of steps required to reach the target state (or the target CLR) which in turn is bounded by $S_{\infty}$. For instance, if we denote by $S_{t}$ the target state, it is easy to see from (2) that the complexity of the algorithm is $O\left(n N\left(S_{t}-S_{0}\right) / \delta\right)$, where $n$ is the number of components in the input vectors and $N$ is the number of small-sized system data. In other words, the complexity of the algorithm, unlike the classic models such as FF, depends only on the FA system parameter. If the CLR is considered as a function of the buffer size, for example, the complexity of the FA algorithm depends only and exclusively on the buffer size, while in the FF or any other analytic model, the complexity depends as well on the number of superposed sources. Note, finally, that the complexity of the FA algorithm can be controlled by the step size $\delta$, a large value of $\delta$ gives a fast but rough approximation of the CLR, while a small value gives a smooth CLR curve but requires longer $\mathrm{CPU}$ times.

To validate our algorithm, in the following, we give some numerical results obtained for the CLR as a function of the buffer size, and the output channel capacity respectively. In each case, we compare our results with the "exact" results provided by alternative methods based on queueing models such as FF or MMDP. The characteristics of the traffic sources used to derive the exact results are depicted in Table I (see [5]). 


\begin{tabular}{cccc}
\hline \hline $\begin{array}{c}\text { Traffic } \\
\text { class }\end{array}$ & $\begin{array}{c}\text { Peak Rate } \\
(\text { Mbits/s) }\end{array}$ & $\begin{array}{c}\text { Mean Rate } \\
(\text { Mbits/s) }\end{array}$ & $\begin{array}{c}\text { Burst Length } \\
\text { (cells) }\end{array}$ \\
\hline \hline Data & 10 & 1 & 339 \\
Image & 2 & 0.087 & 2604 \\
Voice & 0.064 & 0.022 & 58 \\
\hline
\end{tabular}

TABLE I

Traffic Characteristics.

\section{A. CLR as a function of the buffer size}

In this subsection, we apply the FA algorithm to predict the CLR as a function of the buffer size. In this case, it is well known [1], [3] that the asymptotic behavior is governed by

$$
P(m) \asymp L_{0} \exp \left\{\eta S_{m}\right\}, \quad \text { as } S_{m} \rightarrow \infty,
$$

where $S_{m}$ is the buffer size and $\eta$ is the well-known largest negative eigenvalue obtained from the FF approach [1] (see also [3], [19] for heterogeneous traffic). Note that this largest eigenvalue can be expressed in terms of simple traffic parameters such as the first two moments of the burst size and the number of users.

In other words, when $S_{m} \rightarrow \infty$, we have

$$
\log P(m+1)-\log P(m) \asymp \eta\left(S_{m+1}-S_{m}\right)=\eta \delta,
$$

where $\delta$ is the step size from buffer size $S_{m}$ to $S_{m+1}$.

The CLR as a function of the buffer size can sometimes be very small in spite of a small buffer size. This makes it impossible to obtain accurately the small-sized system data from measurements (we suggest later in this section how to solve this problem). On the other hand, the alternative analytical methods often require a large amount of CPU time and memory to solve the most practically interesting cases when the system size is large. Therefore, we suggest the use of the FA algorithm as a complement to these approaches to reduce the computation times and/or avoid the memory problems arising in these approaches. The small-sized system data for example, can be quickly computed from some given analytic model; based on these data, the FA algorithm can be used to obtain a fast and accurate estimation of the CLR for a large-sized system.

To show the accuracy and the effectiveness of the FA algorithm, we use the minimum necessary set of small-sized system inputs. In Figure 3, this number of initial values is equal to five (i.e. $M=5$ ). This means that the fuzzy system is constructed from three input/output pairs. The figure shows the overflow probability as a function of the buffer size for voice calls (cf. Table I). The system load is 0.5 and the number of multiplexed sources is equal

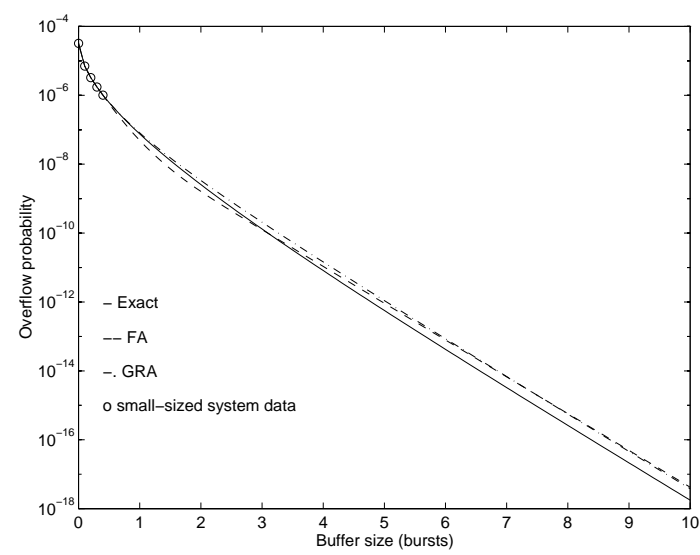

Fig. 3. Overflow probability vs. the buffer size: Markovian sources

to 34 . Note that we chose a relatively small system load to avoid the trivial cases where the curve we want to predict looks more like a straight line (in heavy traffic conditions) than a convex curve.

In this figure, the five input data are obtained from the FF approximation [1] with a step size $(\delta)$ of 0.1 times the mean burst length beginning at zero. The FA algorithm gives a very good approximation compared to the exact results, while requiring ten times less CPU time (10ms) than the GRA approximation (100ms) and about twenty times less than the FF approach (200ms). Besides, the CPU time needed by the FF and the GRA methods can be much larger under other traffic conditions.

Figure 4 shows the overflow probability versus the buffer size for generally distributed burst lengths, as discussed in [3]. The multiplexer buffer is fed now by a superposition of 2-phase Erlang distributed bursts arriving according to a Poisson process. This latter assumption does not restrict the generality of our algorithm. The mean burst size is 2604 cells and the peak rate is equal to $2 \mathrm{Mbits} / \mathrm{s}$. As shown in the figure, the FA algorithm gives a very reasonable approximation. The error is always within one order of magnitude. More numerical examples with other burst length distributions have shown the same accuracy.

In Figure 5 we show the prediction of the overflow probability from a corrupted set of input data with some random errors. This can happen when the set of input data is obtained either from measurements, from a rough simulation or from an inaccurate approximation. The exact curve, is obtained from the exact (FF) curve for a superposition of voice sources with the same traffic parameters as in Figure 3, to which we introduce a small perturbation to the first five data points by adding (or subtracting) a value ranging from $0 \%$ to $50 \%$ respectively to (or from) these points. The smaller is the overflow probability, the larger is the introduced error. In other 


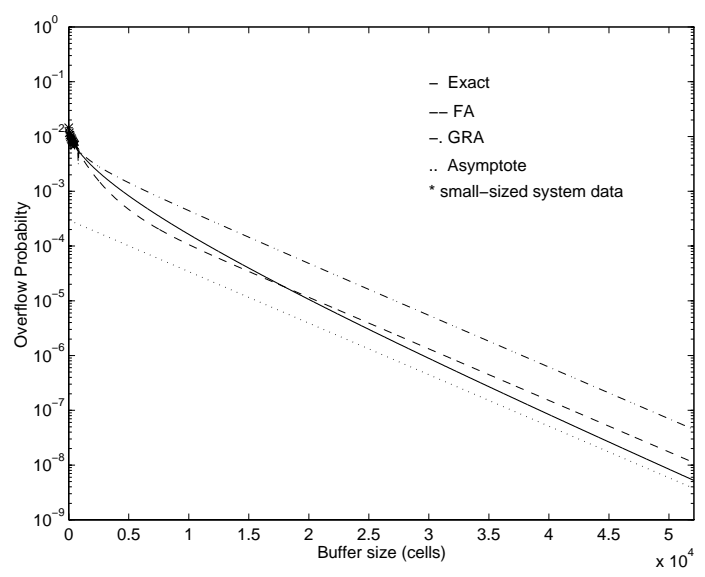

Fig. 4. Overflow probability vs. the buffer size: generally distributed bursts

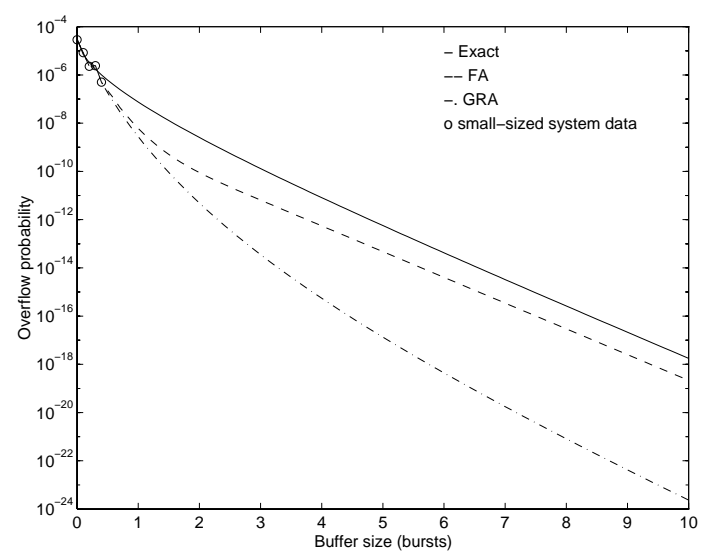

Fig. 5. Overflow probability vs. the buffer size: corrupted input data

words, the first point has no error while the last point can have relative error as large as $50 \%$. This simulates the situation of on-line measurement of real traffic when the measurement time is not very large. Figure 5 shows the accuracy of the FA algorithm in predicting the overflow probability. It is clear that the refinement based on the knowledge of the asymptotic slope is very valuable in this kind of situations.

Figures 4 and 5 show the robustness and the independence of our algorithm from any specific traffic model.

\section{B. CLR as a function of the service capacity}

In this subsection, the FA algorithm is applied to predict the CLR as a function of the service capacity, which is by far the most practically interesting application, mainly because:

- unlike the previous case, the asymptotic behavior in this case depends only on the simplest and the most predictable traffic parameter: the source peak rate. When the service capacity is equal to or larger than the aggregate peak rate

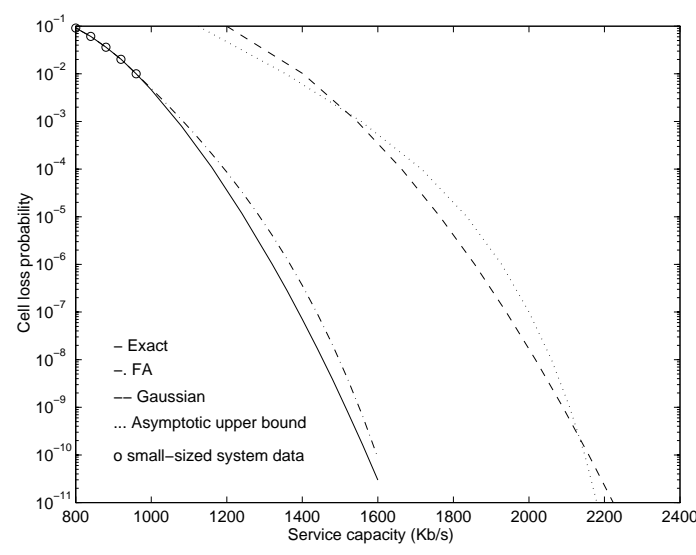

Fig. 6. Cell loss prob. vs. the service capacity for different approaches

of the current connections, then there is no cell loss

$$
P(m)=0, \quad C \geq \text { aggregate peak rate. }
$$

- when the service capacity is small enough, independent of the traffic parameters, the CLR is very high. The small-sized system data can thus be estimated accurately by real-time measurements, without relying on any assumed traffic parameters.

To verify the accuracy of our FA predictions, different numerical examples are shown. Figure 6 shows the cell loss probability as a function of the service capacity. In this figure, the FA estimation is compared with the exact results obtained from the MMDP approximation. In the figure we also show the results obtained from other alternative approximations, namely, the asymptotic upper bound of the CLR [20], [21], given in (4) with $L_{0}=1$, and the stationary Gaussian approximation given in [22]. This latter approximation assumes a zero buffer model and an infinitely large number of connections. The Central Limit Theorem is invoked so that the input rate distribution can be approximated by a Gaussian distribution. The CLR is finally obtained from the tail of the Gaussian approximation. The figure shows the CLR for a superposition of 40 voice sources with traffic characteristics given in Table I. The multiplexer buffer can accommodate 240 cells. We observe that the FA algorithm proves to be much more accurate than the alternative approaches.

Figure 7 shows the FA approximation of the CLR against the service capacity for different numbers of users $N=20$ and $N=40$, with the same characteristics as above. In both cases, the FA algorithm gives accurate estimation of the cell loss probability. Note that the CPU time needed by the FA algorithm is independent of the number of sources. 


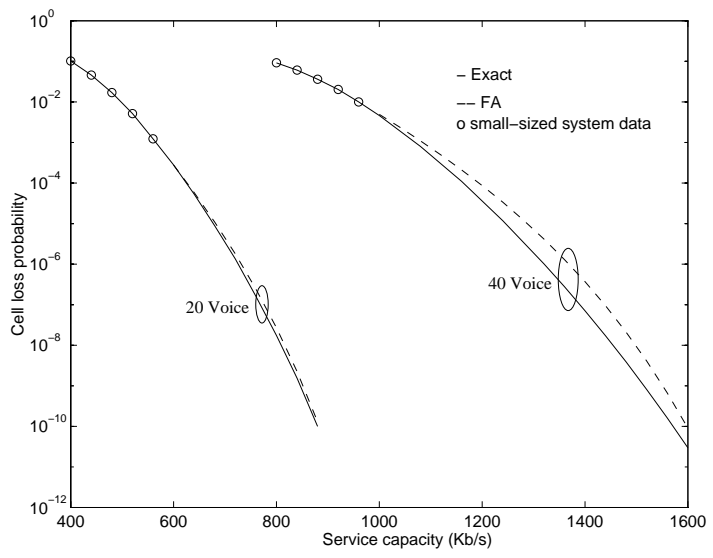

Fig. 7. Prediction of the CLR with homogeneous traffic sources

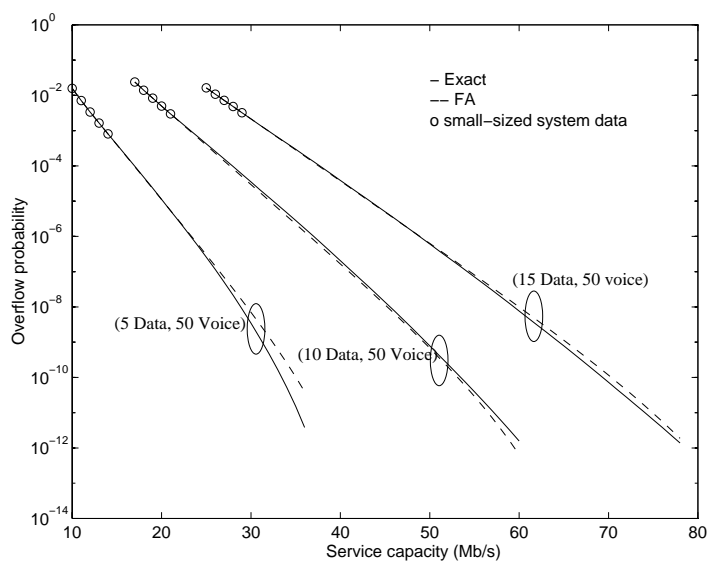

Fig. 8. Prediction of the CLR with heterogeneous sources

The simplicity of this algorithm and its independence of any assumptions on the traffic parameters make it also suitable for the case of heterogeneous traffic, without any modifications. To illustrate this, Figure 8 shows the FA predictions of the overflow probability against the service capacity with three different mixes of heterogeneous sources. The traffic characteristics are given in Table I and the multiplexer buffer can accommodate up to 1695 cells. Again, the FA algorithm predicts accurately the exact values which are obtained from the FF method.

To test the performance of the $\mathrm{FA}$ algorithm when all the traffic characteristics are totally unknown except for the peak rate, Figure 9 shows the FA predictions when the small-sized system data are derived from real-time measurements. The system considered here is a buffer of size 50 cells, served at a service capacity $C$ and fed by a superposition of 10 sources. The traffic characteristics of the sources are all unknown (to the FA algorithm) except the peak rate. The simulation of this system is a real data driven simulation. Each source is an excerpt of approximately ten minutes of MPEG

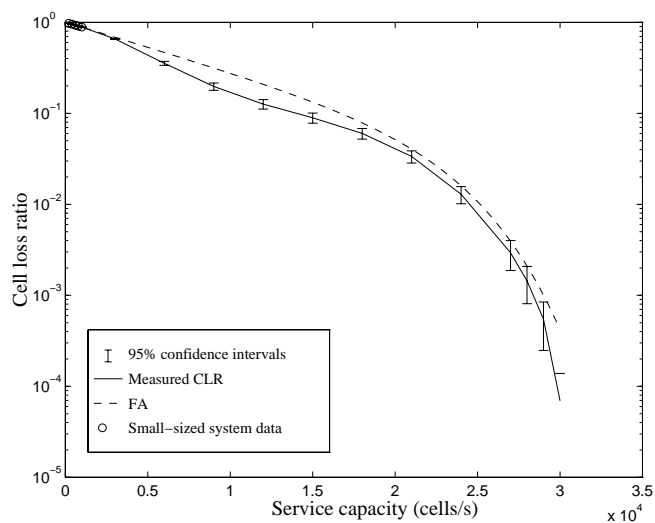

Fig. 9. Prediction of the CLR with measurements on a real video trace

encoded real video source (Star Wars sequence [23], with random phase shift). The simulation assumes these connections are ongoing, and the measurement is stopped after a random time from the start (e.g. corresponding to a call arrival or departure), in this case 40 seconds.

We notice from the results that, when the actual measurements fail to give an accurate estimate of the CLR (i.e. large confidence intervals) for large $C$, the FA estimation continues to decrease regularly. As guaranteed by (6) the CLR will converge to zero when $C$ is equal to the aggregate peak rate. Besides, since the FA algorithm does not depend on traffic parameters such as correlations, we notice that, albeit the traffic sources used in this simulation show a self-similar behavior, as confirmed by Figures 10.a and 10.b, the estimation obtained from the FA algorithm is reasonably accurate even without a prior knowledge or measure of the self-similarity. These two figures show the timevariance plots of the cells inter-arrival times. The abscissa represents the size of the non-interleaving time intervals over which the traffic is aggregated as defined in [24] and the ordinate represents the variance of the resulting cell stream. This shows that our approach is independent of any assumption on the traffic parameters such as the burst length distributions, and more generally the input traffic correlations. Similar results (not shown here) have been obtained when the simulation is driven by independent MPEG sources each being an excerpt of approximately 10 minutes of traffic from a real video sequence (Asterix, News, MTV show, The silence of the lambs, ...).

\section{Extensions and discussions}

As mentioned in Section III-A, it is sometimes difficult to obtain the small-sized system data from measurements when estimating the CLR as a function of the buffer size. If the service capacity is 


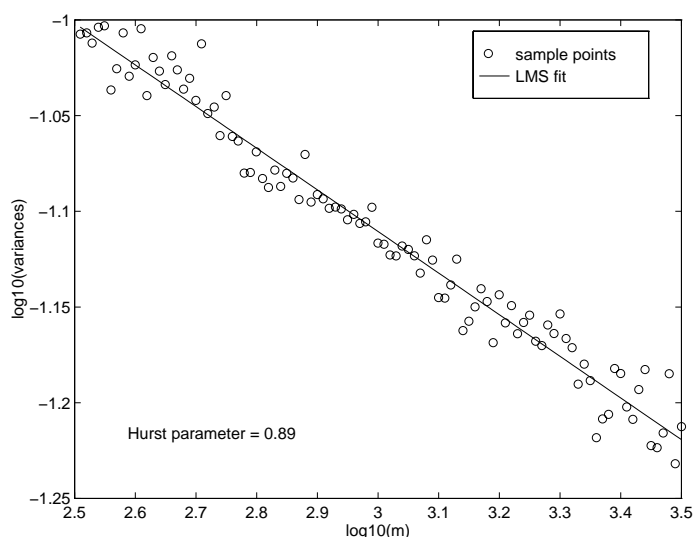

(a) Typical single source

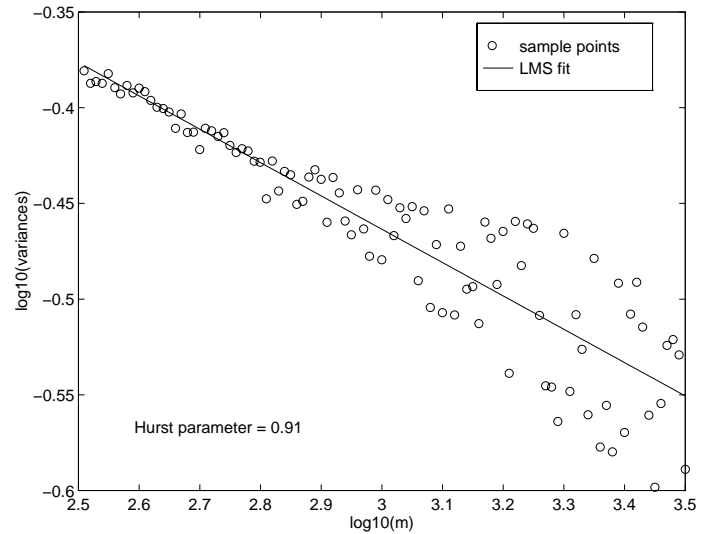

(b) Aggregate traffic

Fig. 10. Time-variance plots of the cell inter-arrival times of a real video trace

high, for example, even when the system is bufferless, the CLR can still be lower than $10^{-6}$. Nevertheless, the flexibility of our FA algorithm allows us to extend it to obtain these measurements accurately. This can be achieved by using our algorithm in two steps: first take measurements with reduced service capacity for each reduced buffer size to estimate the CLR for the target capacity as in Section III-B, and then use the resulting estimations to project the CLR for the target buffer size as in Section III-A. The method can also be applied efficiently to predict the CLR as a function of the number of connected users.

At this stage, we would like to mention that although the predictions obtained from the FA algorithm are accurate, their accuracy is determined largely by the choice of the values of the parameters $S_{\infty}$ and $\sigma$. From extensive numerical tests, we derived a set of heuristic recipes on how to choose these two parameters. For the choice of $S_{\infty}$, the rules are determined intuitively from the asymptotic behavior of the CLR function. For instance,

- for the CLR as a function of the buffer size, $S_{\infty}$ can be chosen equal to ten times the mean burst length when the traffic load is light, while

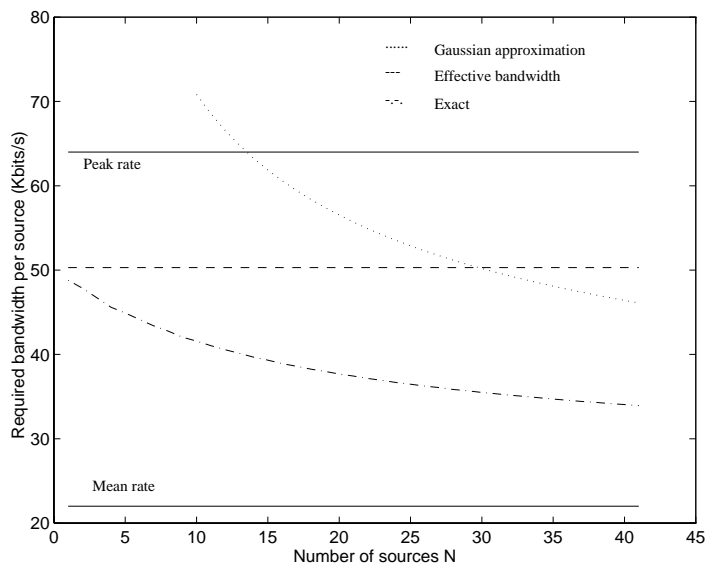

Fig. 11. Comparison of exact values and effective bandwidth approximation

a few (one or two) times the mean burst length are sufficient under heavy traffic conditions;

- for the CLR as a function of the capacity, we have chosen $S_{\infty}$ equal to the aggregate peak rate.

Concerning the value of $\sigma$, up to now, it has been determined by trial and error. For buffer size, we noticed that a value of $\sigma$ equal to $60 \%$ of the value of $S_{\infty}$ is quite reasonable; in the case of the number of users (see [18], [25], the value of $\sigma$ was $48 \%$ of the value of $S_{\infty}$ and finally, in the case of the service capacity, a value of $\sigma$ equal to $28 \%$ of the value of $S_{\infty}$ proves reasonably good. Other methods to determine $\sigma$ are the subject of current research. For instance, our fuzzy system can be combined with other adaptive learning algorithm such as neural networks with off-line simulations to determine an optimal fixed value of $\sigma$. Furthermore, recent suggestions [26] propose to use another filter (3), where $\sigma$ is a function of both $S_{m}$ and $S_{\infty}$ and thus changes dynamically with the value of $S_{m}$.

\section{Application to CAC in ATM Networks}

One of the most important procedures to control congestion in the network is the call admission control (CAC) function. Its goal is to restrict the number of calls simultaneously using the network, in order to provide and ensure QoS to all users in progress. When a new connection request arrives, a contract is established between the source and the network. The source declares a set of traffic parameters to describe its traffic, and the network in turns agrees to guarantee a certain QoS threshold if the source does not violate the declared traffic parameters. If the network has enough resources to accept this additional connection without infringing the QoS of the ongoing connections, the new source is accepted; otherwise, it is rejected.

To allow the network to decide quickly whether a new call can be accepted or not, a fast algorithm 
to estimate either the CLR given the link capacity or the service capacity required by this call to achieve a given CLR is needed. Due to the complex dependence of the CLR on the service capacity and vice versa, the exact required service capacity to guarantee a certain CLR, given the traffic characteristics and the buffer size is very difficult to obtain. Most of the $\mathrm{CAC}$ algorithms proposed in the literature (e.g. [27], [22]) are based on the effective bandwidth concept which computes the required service capacity by approximating the CLR with the asymptotic upper bound given in (4) with $L_{0}=1$. The effective bandwidth concept is attractive because of its additive property: the effective bandwidth for a set of sources is simply the sum of the individual effective bandwidths. This reduces substantially the complexity of the computations. However, due to the infinite buffer size assumption, this approximation does not take into account the statistical multiplexing among the sources sharing the buffer, and sometimes (e.g. when the traffic load is not too heavy) results in an outrageous overestimation of the required service capacity. The stationary Gaussian approximation introduced in Section III-B is also proposed in [22] to take advantage of statistical multiplexing when the number of sources is large. However, it overestimates the required aggregate bandwidth, resulting in an underutilization of expensive network resources such as bandwidth and memory (e.g. see Figure 11). Besides, if the traffic is less bursty than Poisson (e.g. the output of a shaper), it is shown in [28], [29] that the effective bandwidth approximation is no longer conservative and thus results in an underestimation of the required service capacity. More advanced approximations [7], [8] based on the large deviation estimates of the multiplexer queue length distributions often give tighter upper bounds on the CLR than those provided by the effective bandwidth concept. Nevertheless, these approaches still require the complete knowledge of the arrival process to the multiplexer buffer. In view of these problems, we will present below a simple and robust measurement-based CAC mechanism which uses our FA algorithm to estimate the aggregate required bandwidth. For example, for a VBR service, if the bandwidth required by a new call can be roughly estimated, and the total bandwidth used by the ongoing calls can be determined by online monitoring, the network can determine whether it can accept the new call or not. Although our CAC mechanism addresses mainly the VBR service and more generally services with static bandwidth allocation (no bandwidth renegotiation is allowed for the duration of the call), it can be extended easily to include also the RCBR service.

\section{A. The CAC mechanism}

The CAC mechanism we propose comprises two components as shown in Figure 12. The first component consists of a set of virtual buffers (counters) with reduced service capacity to observe very high cell loss with a small variance within a short measurement time interval. Depending on the hardware configuration of the multiplexer, the traffic can be integrated in a common shared buffer or segregated in multiple buffers sharing the same link, in which case as many sets of counters as the number of buffers are needed. The second component of the mechanism consists of the FA algorithm and the decision process. The FA algorithm is used to determine the required bandwidth for the ongoing calls and then the decision process makes the decision to accept or reject the call, based on the outputs of the FA algorithm, the available bandwidth and the bandwidth required by the call. These two parts can be integrated as a single sequential process; however, with regard to hardware implementation issues, we argue that it is better to separate them to minimize the costs and to make the system more modular and thus reusable: for instance the measurement part should run as fast as the multiplexer speed to collect data for measurements, while the FA and decision parts can operate at the end users' time-scale (i.e., call setup time-scale). Besides, depending on the kind of multiplexer used, we may need as many measurement devices as the number of queues that segregate the different traffic classes in the multiplexers, while we need only one device implementing the FA algorithm and the decision procedure. Finally, the FA and decision parts can be moved as well before the switch fabric. In this case, it will be used by all the multiplexer in the switch.

\section{B. The CAC algorithm}

In the following, we present simple diagrams for each part of the CAC algorithm we propose and explain how the different parts interact. Since the three parts of the algorithm are intended to be implemented on separate chips, the corresponding processes are stand-alone and interact with each other through messages. To show this interaction between the three procedures, we adopt the deterministic automata networks to show the interaction between the three processes. The internal behavior of the processes is beyond the scope of this paper. The decision process, the measurement process and the FA procedure are given in Figure 13, Figure 14 and Figure 15 respectively. In these figures, the circles represent the states of the process, the rectangles represent the messages received from another process and the horizontal thick segments represent transitions that are triggered only when the 


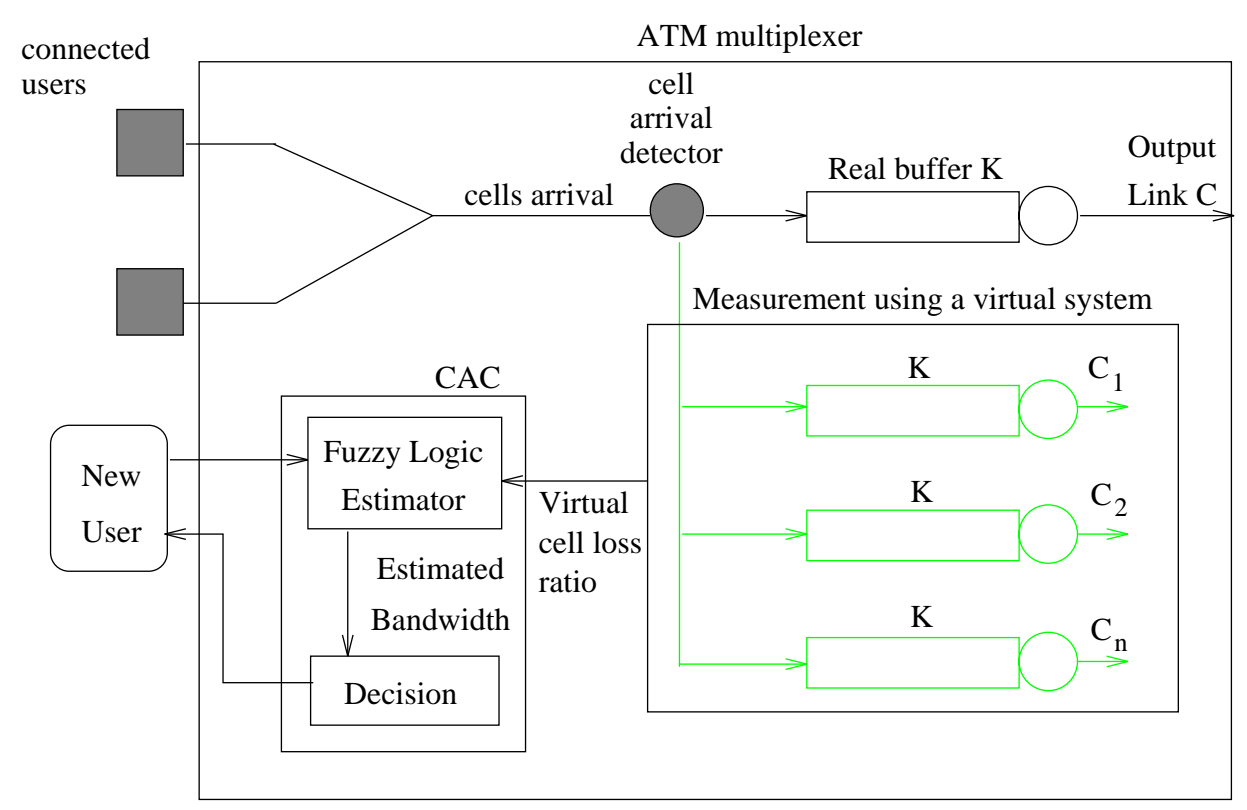

Fig. 12. Fuzzy based CAC mechanism

expected message is received. When a transition is triggered, the process executes the attached list of commands. In the definition of the commands, by convention, we define a label $L$ as a command executed locally, a label $M$ as a message to send to the measurement process, a label $D$ as a message to send to the decision process, and finally a label $F$ as a message to send to the FA process. We denote by $S R B$ the source required bandwidth of the new call, and $B W$ the bandwidth currently used by the ongoing connections. The determination of $S R B$ will be discussed later in this section. We denote by $R B W$ the reserved bandwidth for, say the $N$ ongoing calls: it is defined as the required bandwidth used by the first $N-1$ arrived calls plus the $S R B$ of the last arrived call. We now describe the operation of the decision process and, for the sake of readability of the paper, discuss the operation of the measurement process and the FA process in appendix A.

The decision process controls the starting and ending of the measurement cycles, and makes the decision of accepting or rejecting a call based on the currently used bandwidth $B W$ it receives from the FA process and the estimated value of $S R B$. For instance, in Figure 13, the decision process starts in the WAIT state. Upon the arrival of a connection request, it sends the "Collect measurements 1 " message to the measurement process, sends the value of $S R B$ to the FA process and triggers the transition to go to the "Wait for Bandwidth" state. When in this state, upon reception of the $B W$ value from the FA process, it either accepts the call if
$B W+S R B<C$ and enables the measurement process to start a new measurement cycle through the "Clear measurements" message, or rejects the call if $B W+S R B>C$ and enables the measurement process to return to the WAIT (for a new call) state through the "Restore status" message. Upon a call departure, the decision process sends the message "Collect measurements 2" to the measurement process to enable a new measurement cycle and sends the mean source bandwidth $M S B$ of the departed call to the FA process to enable the update of $B W$ and $R B W$.

As described, the CAC mechanism deals only with those types of services which require a static bandwidth allocation, in the sense of no renegotiation of the bandwidth is allowed during the whole duration of the call. If beside call arrivals and call departures we include the "bandwidth renegotiation" in the class of events that trigger new measurement cycles, the CAC mechanism can be extended to include also the RCBR service. However, in the RCBR service, the bandwidth might be renegotiated in a time-scale much smaller than the duration of a call. In this case, we doubt that our mechanism can be effective as the measurement cycles can be very short and thus may result in inaccurate CLR values.

The determination of the source required bandwidth $S R B$ depends on many factors. First, as we mentioned above, the effective bandwidth can sometimes be non-conservative in which case it underestimates the $S R B$. On the other hand, since the source is not accepted yet, the $S R B$ cannot be 


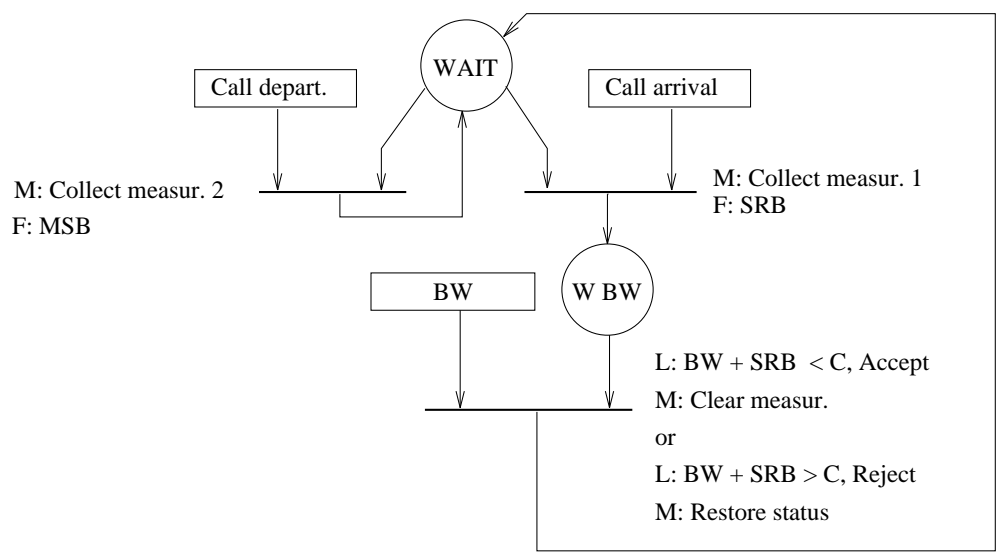

Fig. 13. State diagram of the decision process

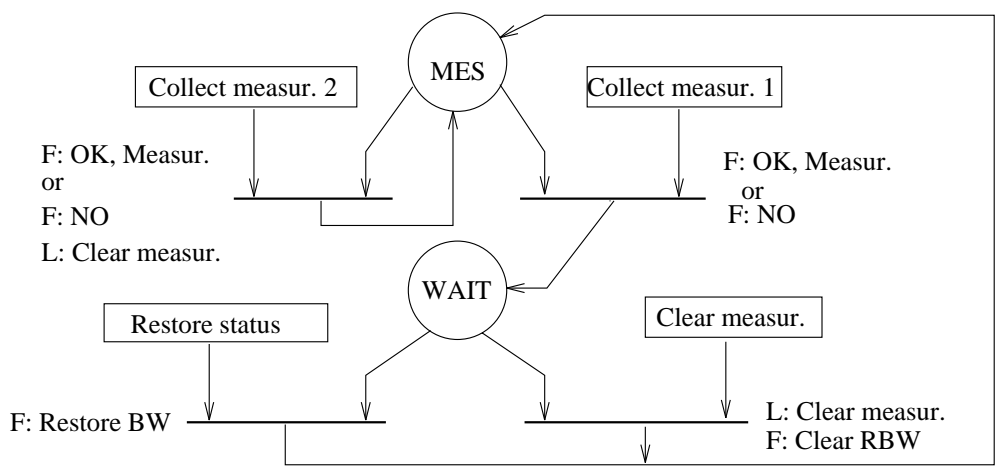

Fig. 14. State diagram of the measurement process

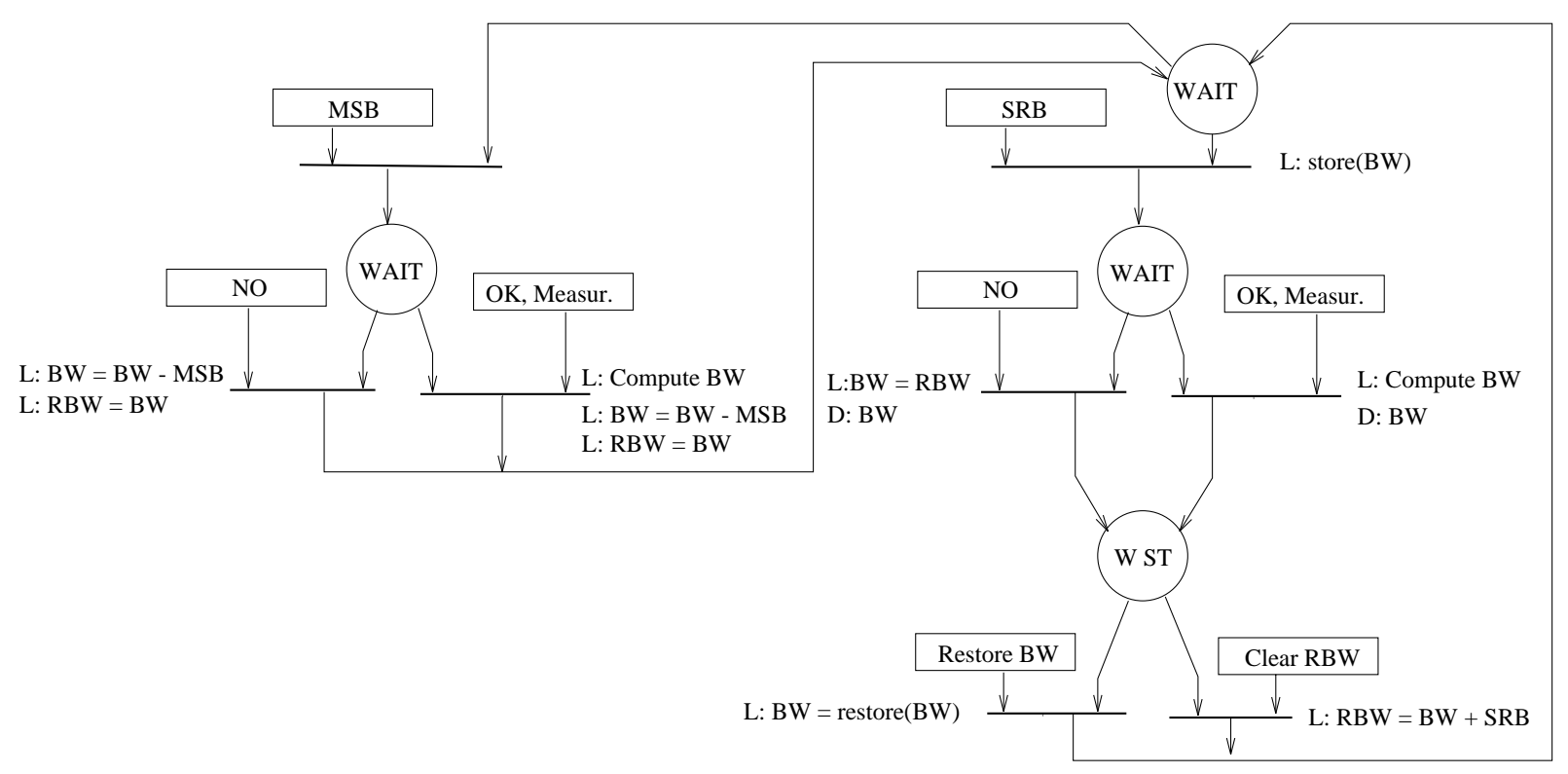

Fig. 15. State diagram of the FA process 
estimated by on-line measurements. Even though this issue is still under investigation, in the following, we give a set of (non exhaustive) guidelines on how to choose the $S R B$ depending on the degree of confidence the network has in the source declared parameters. Assume that the network can classify the traffic sources based on the declared traffic parameters in three categories, "more bursty than Poisson" (MBP), "less bursty than Poisson" (LBP) and "the others" which cannot declare accurate traffic parameters except for the peak rate. For the MBP category, we know that the effective bandwidth is conservative, we thus suggest that the $S R B$ is set to equal the effective bandwidth. Alternatively, for better accuracy, the $S R B$ can also be set equal to the estimate obtained from the large deviation approach in [8]. For the LBP category, either the traffic parameters can be trusted, in which case a simple queueing model such as $\sum D_{i} / D / 1$ or an appropriate queueing model can give the exact value of the $S R B$ for the given CLR, or when the source traffic parameters cannot be trusted, the limiting Poisson arrivals case can give an upper bound on the $S R B$ by solving the $M / D / 1 / K$ queueing system. For the last category, the $S R B$ can simply be set to the source peak rate. In this case, the reserved bandwidth $R B W$ will only slightly overestimate the required bandwidth for one measurement cycle. To justify this, we argue that its impact on the aggregate required bandwidth will be negligible since the bandwidth for the ongoing calls can be estimated accurately with the FA algorithm. Besides, since the reserved bandwidth is updated regularly, when the next call arrives or departs, the FA prediction will allow a dynamic adjustment of the bandwidth. Finally, concerning the source mean bandwidth $M S B$, it can be chosen as the mean source rate when the traffic parameters are known, or can be set to zero when the traffic parameters are unknown. Note that in any case, the $M S B$ should not be larger than the mean source rate. Since our mechanism (unlike the effective bandwidth concept) takes into account the statistical multiplexing gain, the required bandwidth is not additive, so that the departure of one source makes the per-source bandwidth utilization larger. In this case, the mean rate is the only conservative parameter available.

\section{An execution test of the FA CAC mechanism}

To show the gain in bandwidth obtained from our proposed CAC mechanism, we present a simple example of the execution of this algorithm. In this example, we assume homogeneous sources, with the same traffic parameters. Let $\hat{C}$ be the effective bandwidth for one source. We set $S R B=\hat{C}$ and $M S B=\hat{C} / 3$. We assume the effective band- width overestimates the exact required bandwidth by $\hat{C} / 3$. Figure 16 shows the sample path of the reserved bandwidth resulting from our proposed CAC mechanism and compares it to the sample path of the reserved bandwidth resulting from a CAC function based on the effective bandwidth concept: when a new call arrives, $\hat{C}$ is added to the total.

In this figure, we assume $N$ sources are in progress and that the FA estimation of the $R B W$ is as bad as the effective bandwidth initially. Besides, we assume that there is no statistical multiplexing gain: the only gain is obtained from the bad estimation of $S R B$. Figure 16 shows that even under these extreme assumptions, the FA CAC mechanism performs much better than the effective bandwidth concept. We expect even much greater reductions of the reserved bandwidth if we take into account the statistical multiplexing gain.

\section{Conclusions}

In this paper, a new algorithm based on an adaptive fuzzy logic system is proposed to estimate the cell loss ratio in an ATM multiplexer. In this system, the CLR is viewed as a function of a real variable which can be the buffer size, the number of ongoing calls or the multiplexer service capacity. To predict the CLR in the ATM multiplexer with the actual value of the real variable, the fuzzy system does not assume any input traffic model or parameters, however, it relies on the knowledge of a set of CLR values when the variable is small (e.g. small buffer size or small service capacity). This prediction, combined with the knowledge of the asymptotic behavior of the CLR function, allows us to obtain a good approximation of the CLR as a function of the buffer size, the number of users or the multiplexer service capacity. The numerical results have shown that the FA-based estimation of the CLR can give very good approximations while requiring a negligibly small computation time compared to the alternative methods.

To illustrate the efficiency and the practical utility of our algorithm, we have proposed a measurement-based CAC mechanism that uses the FA algorithm to predict the CLR for a given number of connections. Based on this prediction, and the estimated bandwidth required by a new call, an accurate estimation of the aggregate required bandwidth is obtained. This latter is used to decide whether to accept the new call or to reject it.

\section{REFERENCES}

[1] D. Anick, D. Mitra, and M. Sondhi, "Stochastic theory of a data handling system with multiple sources," Bell Systems Technical Journal, vol. 61, no. 8, pp. 18711894, 1982.

[2] I. Norros, J. W. Roberts, A. Simonian, and J. Virtamo, "The superposition of variable bitrate sources in 
on Communications, vol. 44, pp. 203-217, February 1996.

\section{Appendix A}

Operation of the measurement process

The measurement process is constantly monitoring the number of cell arrivals and the number of cell losses in the virtual buffers with down-sized capacity. When it receives a "Collect measurements" message, it computes the CLR and the confidence interval of the CLR. If the confidence intervals are in a predefined "trustable" range, it sends the message "OK" to the FA process followed by the obtained values of the CLR in the virtual buffers. Otherwise, it assumes the measurements are not accurate enough and notifies the FA process through the message "NO". It then triggers the corresponding transition and waits for a message from the decision process to either clear the measurements (the call has been accepted) or restore its internal status (the call has been rejected). In the first case, the measurement process also notifies the FA process to clear the old value of $R B W$, while in the second case it enables the FA process to restore the old value of $B W$.

\section{Operation of the FA process}

In addition to the FA algorithm proposed in this paper, the FA process contains other functions to interact with the other parts of the CAC algorithm. The FA process is initially in a standby state waiting for messages from the decision process. When it receives the "SRB" message containing the source required bandwidth value $S R B$, it triggers the transition and stores the old value of the required bandwidth $B W$. It then waits for the status of the measurements from the measurement process. If it receives the status message "NO" meaning that the measurements are not accurate, it just sends the reserved bandwidth $R B W$ for the ongoing calls to the decision process. If it receives the status message "OK" meaning that the measurements are obtained with confidence, it transits to a state where it waits for the measurement values. Upon reception of the measurement values, it then computes the required bandwidth $B W$ using the FA algorithm and sends the result to the decision process. In either case, it transits to the state W STAT where it waits for the status of the connection request: if the connection has been accepted (i.e. "Clear $R B W$ " message is received), the FA process computes the new reserved bandwidth $R B W=B W+S R B$ and then transits to the initial state; if the connection has been rejected (i.e. "Restore BW" message is received), the old value of $B W$ is restored. Upon a call departure, the FA process receives the value of the $M S B$ (mean source bandwidth) from the deci- sion process. In this case, the FA process triggers immediately the corresponding transition and waits for the status of the measurements from the measurement process. If the measurements are not accurate (the status message "NO" is received), then both $B W$ and $R B W$ are set to $B W-M S B$. If the measurements are accurate, the status message "OK" followed by the measured CLR values are received from the measurement process. In this case, the FA algorithm is used to estimate the value of $B W$ including the connection which has departed, and $B W$ and $R B W$ are set to $B W-M S B$. 